\title{
Dynamic Colonization of Gut Microbiota and Its Influencing Factors Among The Breast-Feeding Infants During The First Two Year of Life
}

\section{Ping Li}

Capital medical university https://orcid.org/0000-0002-1310-1092

\section{Xuelian Chang}

Capital Medical University

\section{Xiaoyu Chen}

Capital Medical University

Tiantian Tang

Capital Medical University

Yajing Liu

Capital Medical University

Yu Shang ( $\nabla$ shangyuhao@163.com )

Capital Medical University

Kemin Qi

Capital Medical University

\section{Research}

Keywords: Maturation, gut microbiota, BMl, faeces, break milk

Posted Date: July 27th, 2021

DOI: https://doi.org/10.21203/rs.3.rs-717538/v1

License: (c) (i) This work is licensed under a Creative Commons Attribution 4.0 International License. Read Full License 


\section{Abstract \\ Background}

Maturation of the infant gut microbiota has lifelong implications on health, which has been proposed as the major events during the first year of life. However, little was known about dynamic colonization of the gut microbiota and its influencing elements among the two-year infancy as well as into the adulthood.

\section{Results}

Based on the 16S rRNA sequencing data in the V3-V4 regions among 30 healthy mother-infant pairs with the normal range of the growth and development index from birth to two years old, the diversity of the gut microbiota was significantly increased from Six-month to Two-year subgroups. Furthermore, the dynamic colonization of gut microbiota was that the significant trends of Firmicutes (Faecalibacterium, Blautia, Enterococcus, Subdoligranulum, Agathobacter, Unidentified_Erysipelotrichaceae, Staphylococcus, Acinetobacillus, Unidentified_Ruminococcaceae and Fusicatenibacter), Bacteroidetes and Verrucomicrobia were increased, while Actinobacteria (Bifidobacterium) and Proteobacteria (Enterobacteriaceae and Klebsiella) were decreased with the increased age at the phylum and genus levels. These above results revealed that certain bacteria might modulate the host pathways, such as Chemoheterotrophy, Fermentation, Parasites_or_symbionts, Nitrate_reduction and Aerobic_chemoheterotrophy metabolism. Moreover, there were significant associations between maternal (gut microbiota in the milk, Pre-pregnancy BMI-M.BMI, BMI gain during the pregnancy-I.BMI) and infant characteristics (BMI at birth-B.BMI and increment of BMI-G.BMI), and the compositions of gut microbiota in the faeces, but not differences were shown between the different sex and mode of productive subgroups.

\section{Conclusion}

Overall, the gut microbial community was significantly matured into adulthood with the increased age subgroups. It also identified that there were significant correlations between the features of gut microbiota and maternal (gut microbiota in the milk, M.BMI and I.BMI) and infant characteristics (B.BMI and G.BMI), which will provide a new direction for the host-gut microbiota interplay during the two years of life.

\section{Background}

The human gut ecosystem is composed of $10^{11}$ to $10^{12}$ micro-organisms per gram in the feces, and estimates to harbor more than 500 bacterial species, which is a large and diverse community of microorganisms in the close cross-talk with its host [1]. As we all known, the gut microbiota is an important environmental factor for human health, having evolutionarily conserved roles in the 
metabolism, immunity, development, behavior of the host and other conditions[2]. Although considerable efforts have focused on cataloguing the adulthood gut microbiome and its relationship to complex diseases. The studies on the infant gut microbiota have been restricted to the cooperative and competitive interactions between the bacteria within one year of life [3]. It is important, therefore, to understand how gut microbiotas are established and the related elements that result in the stable gut microbiota.

Recently, some researches have proved that early infancy is a key period in the development and colonization of gut microbiota, which represents the pivotal de novo assembly of a complex microbial community from an ecological point of view thought to begin at birth and affects the later-life health [4, 5]. Exactly, after weaning, the infant gut microbiota stabilizes to the adult form by around the age of 2 years when it settles into an adult-like anaerobic pattern. It is agreement with the changes of diet type among the Chinese infants, whose energy sources were respectively from the pure breast feeding at 6 months, initial solid food introduction at one year and the diet tendency to the adults at two years old [6]. Presently, more studies focus on the colonization of intestinal flora and its influencing factors within first one year of life [1-5], but little is known about the transfer of gut microbiota on the whole life cycle including the age of two years old.

As many previous studies have confirmed that the colonization and pattern of infant gut microbiota is a complex process. It depends on multiple overlapping environmental and host factors, such as the delivery mode, birth hospitalization, compositions of the maternal gut microbiota in the breast milk, vaginal or skin, type of infancy feeding, weight at birth, gestational age at birth, and prenatal administration of the probiotics and intrapartum antibiotics prophylaxis [7-10]. However, it is refuted whether and how the implication of these above factors exert long-term effects on the abundance of specific gut bacterial in some studies [9-12]. So far, there is insufficient explanation regarding the pattern and diversity of infant gut microbiota in the relation to the known influencing factors. To better elucidate this relation, we performed 16S rRNA sequencing in the breast milk and fecal samples from 30 full-term mother-infant pairs with the normal range of the growth and development index (BMI, LAZ, WAZ and BMI Z: -1 to 1) at different ages (at birth, 6 months, one year, one and a half year, and two years) from birth to two years and then demonstrated the gut microbiome related signatures of maternal and infants' characteristic to drive the changes in the compositions of gut microbiota in the present birth cohort among Chinese Han population of exclusive breastfeeding within 6 months at the First Hospital of Tsinghua University during the two year of life by adjusting the confounding factors, which may constitute an important research tool on providing a new direction for host-gut microbiota interplay.

\section{Results}

\section{Characteristics of the study population}

We randomly enrolled 30 healthy mother-infant pairs in this study by following up to two years old with the normal range of the growth and development indexes (BMI, LAZ, WAZ and BMI Z: - 1 to 1) at birth, 6 
months, one year, one and a half year, and two years respectively at the First Hospital of Tsinghua University (Fig. 1). The characteristics of the study population was shown in Table 1, the infants were born at the gestational age (38.91 \pm 1.35 week), in which $18(60.00 \%)$ subjects were produced by the cesarean and 17 (56.67\%) were boy. Meanwhile, the mother age at the time of specimen collection was $(31.09 \pm 3.44)$ year with the $(12.98 \pm 3.07) \mathrm{kg}$ weight and $(4.91 \pm 1.15) \mathrm{kg} / \mathrm{m}^{2} \mathrm{BMl}$ gain during the whole pregnancy. Moreover, Table 1 demonstrated there were no significant differences of these characteristics at baseline including the maternal age, height, pre-pregnancy weight, pre-pregnancy BMI, prenatal weight, prenatal BMI, weight gain, BMI gain, gestational age, sex of the infants and mode of delivery between all subjects $(n=254)$ and the enrolling 30 healthy mother-infant pairs $(n=30)$ in this research $(P>0.05)$.

Table 1

Descriptive data of the basic information in this study $(n=30)$

\begin{tabular}{|c|c|c|c|c|}
\hline Index & $\begin{array}{l}\text { All subjects }(n= \\
254)\end{array}$ & $\begin{array}{l}\text { Enrolling subjects }(n= \\
\text { 30) }\end{array}$ & $t / x^{2}$ & $P$ \\
\hline Pregnant woman & $31.88+3.97$ & $31.09+3.44$ & 0.363 & 0.729 \\
\hline \multicolumn{5}{|l|}{ Age (years) } \\
\hline Height (cm) & $162.32+7.86$ & $162.58+14.52$ & 0.027 & 0.980 \\
\hline Pre-pregnancy weight $(\mathrm{kg})$ & $57.91 \pm 11.11$ & $55.54+6.07$ & 0.460 & 0.662 \\
\hline Pre-pregnancy BMI $\left(\mathrm{kg} / \mathrm{m}^{2}\right)$ & $21.68 \pm 3.76$ & $21.01+2.77$ & 0.351 & 0.738 \\
\hline Prenatal weight $(\mathrm{kg})$ & $70.81 \pm 9.06$ & $68.52+6.54$ & 0.484 & 0.645 \\
\hline Prenatal BMI $\left(\mathrm{kg} / \mathrm{m}^{2}\right)$ & $26.55 \pm 2.77$ & $25.92+3.10$ & 0.344 & 0.742 \\
\hline Weight gain $(\mathrm{kg})$ & $12.91 \pm 3.36$ & $12.98+3.07$ & 0.035 & 0.973 \\
\hline BMI gain $\left(\mathrm{kg} / \mathrm{m}^{2}\right)$ & $4.87 \pm 1.37$ & $4.91+1.15$ & 0.127 & 0.903 \\
\hline Infant & $39.08+1.40$ & $38.91+1.35$ & 0.251 & 0.810 \\
\hline \multicolumn{5}{|l|}{ Gestational age (week) } \\
\hline Sex-boy (\%) & $132(51.97 \%)$ & $17(56.67 \%)$ & 0.237 & 0.626 \\
\hline $\begin{array}{l}\text { Mode of production-Natural } \\
(\%)\end{array}$ & $161(63.39 \%)$ & $18(60.00 \%)$ & 0.132 & 0.716 \\
\hline
\end{tabular}

\section{Diversity of the gut microbiota between the different age subgroups}

To characterize the dynamic colonization of intestinal flora at different ages, we shotgun the sequenced stool samples at 6 months, one-year and two years by 16SrRNA sequencing in the V3-V4 region. As 
shown in the results of Fig. 2A and 2B, the diversity of the gut microbiota was significantly increased with the age (Two-year group vs One-year group, Two-year group vs Six-month group, One-year group vs Sixmonth group), which was expressed by the Shannon and Simpson index $(P<0.05)$. However, no significant difference in the richness of the gut microbiota (Chao and Ace index) was found among the different age subgroups of 6 months, one year and two-years old ( $(P>0.05$, Fig. 2C and 2D). To assess the overall structure of gut microbiota, the score plots of non-metric multidimensional scaling (NMDS) based on unweighted UniFrac distances were constructed. The results in Fig. 2E, 2F and 2G showed that the structures and compositions of the gut microbiota successfully partitioned into three distinct sections from the phylum and genus levels at 6 month, one-year and two-year subgroups. It was also demonstrated by the network ternary-plot in Fig. $2 \mathrm{H}, 2 \mathrm{I}$ and $2 \mathrm{~J}$ at 6 month, one-year and two-year subgroups from the phylum to genus levels $(P<0.05)$.

\section{Dynamic colonization of the changes in the gut microbiota between the different age subgroups}

We further performed the dynamic colonization of the changes in the gut microbiota between the different age subgroups at the phylum (Fig. 3A) and genus levels (Fig. 4A and 4B). From the phylum level in Fig. 3B, Actinobacteria constituted the dominant phylum in the infant stool at the Six-month group, while Firmicutes was the dominant phylum in the infant faeces at one-year and two-year groups $(P<$ 0.05). However, there was no significant difference at the percents of Bacteroietes among six-month $(2.65 \pm 1.72) \%$, one-year $(1.54 \pm 1.67) \%$ and two-year groups $(3.21 \pm 4.05) \%(P>0.05)$. Exactly, the Firmicutes ( $21.77 \%$ vs $41.67 \%$ vs $65.27 \%$ ) (Fig. 3 C), Bacteroidetes ( $2.65 \%$ vs $1.54 \%$ vs $3.21 \%$ ) (Fig. 3 F) and Verrucomicrobia ( $0.019 \%$ vs $0.18 \%$ vs $0.45 \%$ ) (Fig. $3 G$ ) were increased, while the compositions of Actinobacteria ( $38.44 \%$ vs $30.26 \%$ vs $23.49 \%$ ) (Fig. 3D) and Proteobacteria (36.92\% vs $26.25 \%$ vs $7.45 \%$ ) (Fig. 3E) were decreased from Six-month to Two-year subgroups (Six-month group vs One-year group vs Two-year group) in Fig. 3H $(P<0.05)$.

To structurally organize and taxonomically annotate the gut microbiota from the genus levels at the sixmonth, one-year and two-year subgroups (Fig. 4C and 4D), the gut microbiota of Bifidobacterium, Enterobacteriaceae, Klebsiella and Veillonella were much lower, and higher compositions of unidentified_Lachnospiraceae, Faecalibacterium, Blautia, Enterococcus, Subdoligranulum, Agathobacter, Erysipelotrichaceae, Staphylococcus, Acinetobacillus, Ruminococcaceae and Fusicatenibacter in the Two-year group than those in the Six-month and/or One-year subgroups $(P<0.05)$. While lower levels of Bifidobacterium, Enterobacteriaceae and Klebsiella, with higher levels of unidentified_Lachnospiraceae, Faecalibacterium, Veillonella, Blautia, Subdoligranulum, Agathobacter, Unidentified_Erysipelotrichaceae, Unidentified_Ruminococcaceae and Fusicatenibacter in the One-year group than those in the six-month group $(P<0.05)$. However, there was no significant difference at the percent of Lactobacillus among sixmonth $(1.34 \pm 0.40) \%$, one-year $(1.21 \pm 0.15) \%$ and two-year subgroups $(0.90 \pm 0.18) \%(P>0.05)$.

Moreover, the significant trends of gut microbiota at the genus level were that the percent of Bifidobacterium( $36.93 \%$ vs $28.94 \%$ vs $21.92 \%$ ), Enterobacteriaceae ( $26.03 \%$ vs $21.06 \%$ vs $4.94 \%$ ) and Klebsiella (6.98\% vs $1.55 \%$ vs $0.26 \%)$ were significantly decreased, while Faecalibacterium $(0.40 \%$ vs 
$3.38 \%$ vs $6.47 \%$ ), Blautia ( $0.65 \%$ vs $2.79 \%$ vs $8.29 \%$ ), Enterococcus $(0.48 \%$ vs $0.40 \%$ vs $2.94 \%)$,

Subdoligranulum ( $0.12 \%$ vs $1.33 \%$ vs $2.61 \%$ ), Agathobacter ( $0.15 \%$ vs $0.72 \%$ vs $4.93 \%$ ), Unidentified_Erysipelotrichaceae (0.09\%vs $0.48 \%$ vs $2.85 \%)$, Staphylococcus $(0.015 \%, 0.023 \%, 0.53 \%)$, Acinetobacillus $(0.012 \%, 0.019 \%, 0.51 \%)$, Unidentified_Ruminococcaceae $(0.21 \%$ vs $0.82 \%$ vs $2.52 \%)$ and Fusicatenibacter $(0.12 \%$ vs $0.79 \%$ vs $3.32 \%)$ was increased from six months to two years (Six-month vs One-year vs Two-year group) (Fig. 4E) $(P<0.05)$.

\section{Functional maturation of the gut microbiota}

To determine the functional capacity and trends of the infants gut microbiota developed during the first two year of life, we analyzed the functional maturation of gut microbiota using the KEGG pathways. As shown in Fig. 5B, the gut microbiome evolved into the more complex and adult like configuration in the two-year group, which was significantly reduced inter individual differences than those in the six-month and one-year groups $(P<0.05)$. As the result of the functional maturing infant gut microbiota in Fig. 5A and $5 \mathrm{C}$, we observed that the functional maturation of gut microbiomes was enriched in Chemoheterotrophy, Fermentation, Animal_parasites_or_symbionts, Human_gut, Mammal_gut, Nitrate_ reduction, Aerobic_chemoheterotrophy, Human_pathogens_all and Human_pathogens_diarrhea at the six-month, one-year and two-year subgroups, which was consisted with more than $75 \%$ gut microbiomes in the faeces $(P<0.05)$. Comparing with the succession of bacterial metabolic function in the Two-year group, Chemoheterotrophy, Fermentation and Animal_parasites_or_symbionts were all higher in the Sixmonth and One-year groups, which were more than $50 \%$ microbiomes in the faeces $(P<0.05)$. Meanwhile, all intestinal flora related functions in the One-year group was in agreement with those in the Six-month group $(P>0.05)$.

\section{Associations between the a-diversity and compositions of the gut microbiota over the first two-year of the life}

In the regression models (shown in Table 2), the gut microbiota in the stool samples was negatively associated with the significant differences in shannon and simpson at 6 month $(r=-0.495, P=0.0120$; $r=-0.639, P<0.001)$, one-year $(r=-0.774, P<0.001 ; r=-0.746, P<0.001)$ and two-year $(r=-0.740, P=0.00105$; $r=-0.792, P<0.001)$, while the positive correlations were shown between the shannon index at 6 month $(r$ $=0.455, P=0.0222)$ and one-year $(r=0.445, P=0.432)$ with the maternal BMI increment during the whole pregnancy. 
Table 2

Rank-based regression models between the alpha diversity and compositions of the gut microbiota at different age groups $(r, \mathrm{P})$

\begin{tabular}{|c|c|c|c|c|}
\hline Index $(n=30)$ & Shannon & Simpson & Chao & ACE \\
\hline \multicolumn{5}{|l|}{ Six-month } \\
\hline Gut microbiota in the milk & $-0.495,0.012$ & $-0.639,<0.001$ & $0.181,0.387$ & $0.124,0.555$ \\
\hline BMI at six-month & $0.078,0.712$ & $-0.220,0.291$ & $0.288,0.162$ & $0.279,0.177$ \\
\hline BMI at birth & $-0.160,0.445$ & $-0.237,0.254$ & $0.175,0.404$ & $0.103,0.624$ \\
\hline BMI increment of the infant & $0.302,0.143$ & $0.172,0.410$ & $0.089,0.671$ & $0.142,0.500$ \\
\hline Gestational weeks & $-0.055,0.795$ & $0.071,0.735$ & $0.031,0.886$ & $0.020,0.924$ \\
\hline Maternal age at pregnancy & $0.112,0.596$ & $0.325,0.113$ & $-0.259,0.212$ & $-0.196,0.348$ \\
\hline $\mathrm{BMI}$ at the pre-pregnancy & $0.004,0.984$ & $0.159,0.448$ & $0.037,0.859$ & $-0.005,0.981$ \\
\hline $\begin{array}{l}\text { BMI increment during the } \\
\text { whole pregnancy }\end{array}$ & $0.455,0.022$ & $0.206,0.323$ & $0.394,0.051$ & $0.406,0.044$ \\
\hline \multicolumn{5}{|l|}{ One-year } \\
\hline Gut microbiota in the milk & $-0.774,<0.001$ & $-0.746,<0.001$ & $0.014,0.951$ & $0.076,0.743$ \\
\hline BMI at one-year & $0.073,0.754$ & $0.177,0.444$ & $0.167,0.468$ & $0.186,0.420$ \\
\hline BMl at birth & $-0.096,0.679$ & $-0.113,0.626$ & $0.226,0.325$ & $0.261,0.253$ \\
\hline BMI increment of the infant & $-0.021,0.929$ & $0.103,0.658$ & $-0.183,0.427$ & $-0.203,0.378$ \\
\hline Gestational weeks & $0.174,0.452$ & $-0.102,0.659$ & $0.366,0.103$ & $0.423,0.0559$ \\
\hline Maternal age at pregnancy & $0.151,0.515$ & $0.185,0.421$ & $-0.066,0.775$ & $-0.031,0.894$ \\
\hline $\mathrm{BMI}$ at the pre-pregnancy & $0.222,0.335$ & $-0.094,0.687$ & $0.213,0.354$ & $0.238,0.299$ \\
\hline $\begin{array}{l}\text { BMI increment during the } \\
\text { whole pregnancy }\end{array}$ & $0.445,0.043$ & $0.335,0.137$ & $0.417,0.060$ & $0.424,0.056$ \\
\hline \multicolumn{5}{|l|}{ Two-year } \\
\hline Gut microbiota in the milk & $-0.740,0.001$ & $-0.792,<0.001$ & $0.033,0.905$ & $-0.128,0.638$ \\
\hline BMI at two-year & $-0.044,0.871$ & $-0.103,0.704$ & $-0.050,0.854$ & $0.027,0.922$ \\
\hline BMl at birth & $0.159,0.557$ & $0.179,0.506$ & $-0.171,0.528$ & $-0.138,0.610$ \\
\hline BMI increment of the infant & $-0.246,0.359$ & $-0.306,0.249$ & $0.081,0.766$ & $0.146,0.591$ \\
\hline Gestational weeks & $-0.061,0.824$ & $0.012,0.964$ & $0.051,0.851$ & $-0.068,0.801$ \\
\hline
\end{tabular}




\begin{tabular}{|lllll|}
\hline Index $(\mathbf{n}=\mathbf{3 0})$ & Shannon & Simpson & Chao & ACE \\
\hline Maternal age at pregnancy & $-0.047,0.862$ & $-0.025,0.926$ & $-0.208,0.439$ & $-0.120,0.659$ \\
\hline BMI at the pre-pregnancy & $-0.474,0.064$ & $-0.400,0.135$ & $-0.459,0.074$ & $-0.547,0.028$ \\
\hline BMI increment during the & $-0.362,0.169$ & $-0.418,0.107$ & $0.224,0.405$ & $0.153,0.572$ \\
whole pregnancy & & & & \\
\hline
\end{tabular}

\section{Associations between quantitative characteristics and the compositions of the gut microbiota in the infant ' faeces over the first two-year of the life}

The compositions of the infant gut microbiota in the faeces over the first two-year of the life at the phylum and genus levels (Fig. 6) showed some associations with the maternal and infant characteristics. As shown in Fig. 6A and Fig. 6B when the infants were 6 months old, the compositions of gut microbiomes (Actinobacteria and Cyanobacteria-positively, Firmicutes and undentlfied_Bacteria negatively) were significantly associated with that in the breast milk (GM) $(P<0.05)$, while the BMI at birth (B.BMI) could affect the percents of Firmicutes (negatively), Actinobacteria (positively) and Verrucomicrobia (positively) $(P<0.05)$, and the percent of Tenericutes was negatively correlated with the $\mathrm{BMI}$ at the 6 months old (S.BMI) and positively correlated with the maternal pre-pregnancy BMI (M.BMI) in the faeces at the phylum level (Fig. 6A) $(P<0.05)$. What is more, positively correlation between the composition of Chloroflexi and gestational week $(\mathrm{G})$, negatively correlation between the composition of Undentlfied_Bacteria and maternal age (MA) $(P<0.05)$, and positively correlations between the composition of Bacteroidetes and $\mathrm{BMI}$ increment during the pregnancy (I.BMI) were also demonstrated in Fig. 6A $(P<0.05)$. While at the genus level, the compositions of gut microbiomes (Bifidobacterium and Rothia-positively, undentlfied_Lachnospiraceae, Blautia, Delftia and undentlfied_Erysipelotrichaceaenegatively) were significantly associated with the GM $(P<0.05)$, while the compositions of Bacteroidetes, Collinsella and Flavonifractor were positively correlated with that in I.BMI $(P<0.05)$. And the percent of gut microbiota were negatively associated with the MA (Veillonella, Granulicatella and Subdoligranulum) and M.BMI (Blautia, Faecalibacterium and Agathobacter) $(P<0.05)$. What is more, B.BMI could affect the percents of Bifidobacterium (positively), Rothia (positively) and undentlfied_Lachnospiraceae (negatively) in the Fig. 6B $(P<0.05)$.

When the subjects were one year old, the compositions of gut microbiomes at the phylum level (Firmicutes-negatively and Actinobacteria-positively) were significantly associated with $\mathrm{GM}(P<0.05)$, while there were positive correlations between maternal and infant characteristics and the compositions of the gut microbiota in the infant faeces (Fusobacteria and B.BMI, Verrucomicrobia and G) $(P<0.05)$, which were also shown of the negative correlations (Fusobacteria and I.BMI, Chloroflexi and G. BMI) in the faeces (Fig. 6C) $(P<0.05)$, while at the genus level, the compositions of gut microbiomes (Bifidobacterium, Klebsiella, Streptococcus, Enterococcus and Rothia-positively, Blautia, Hungatella, Undentlfied_Lachnospiraceae, Undentlfied_Erysipelotrichaceae and Anaerostipes-negatively) were 
significantly associated with that in GM $(P<0.05)$, while the compositions of Klebsiella and Fusobacterium were negatively correlated with the I.BMI, Agathobacter and Butyricicoccus were positively associated with M.BMI $(P<0.05)$. What is more, B.BMl could affect the percents of Fusobacterium (positively), Subdoligranulum (positively) and Undentlfied_Clostridiales (negatively) $(P<0.05)$.

Furthermore, the compositions of gut microbiomes (Agathobacter, Akkermansia, Collinsella, Undentlfied_Ruminococcaceae, Fusicatenibacter and Butyricicoccus- positively, Megasphaera and Lachnoclostridium-negatively) were significantly associated with that in the $\mathrm{G}$ in the Fig. $6 \mathrm{D}(P<0.05)$.

When the subjects were two years old, the compositions of gut microbiomes at the phylum level (Firmicutes and Bacteroidetes- negatively, Actinobacteria and Proteobacteria-positively) were significantly associated with the $\mathrm{GM}$, while the $\mathrm{G}$ and $\mathrm{M}$.BMI could respectively affect the percents of Tenericutes and Cyanobacteria in the faeces (Fig. 6E) $(P<0.05)$. From the genus level, there were positively correlations between the maternal and infant characteristics and the compositions of the gut microbiota (GM and Bifidobacterium/Undentlfied_Enterobacteriaceae, B.BMI and Faecalibacterium/Roseburia, G.BMI and Undentlfied_Rhizobiaceae, M.BMI and Streptococcus) $(P<0.05)$, which were also proved the negative associations (GM and

Bacteroidetes/Subdoligranulum/Fusicatenibacter/Undentlfied_Erysipelotrichaceae/

Undentlfied_Ruminococcaceae/Parabacteroides/Lachnospira/Sarcina/Dorea/Butyricicoccus, T.BMI and Romboutsia/Undentlfied_Prevotellaceae, G.BMI and Blautia/ Roseburia, M.BMI and Undentlfied_Lachnospiraceae/Romboutsia/Butyricicoccus, I.BMI and Anaerostipes) in the faeces (Fig. 6F) $(P<0.05)$.

\section{Effects of qualitative characteristics on the profiles of faecal gut microbiota in the infants}

Significant shifts in the composition of profiles of faecal gut microbiota in the infants were observed through stratified intervention including the sex (Fig. 7) and mode of production (Fig. 8) using a permutational ANOVA of unweighted and weighted Unifrac distance in this birth cohort. Concretely, the adiversity index (Shannon, Simpson, Chao and ACE, Fig. 7A), $\beta$ diversity index (PCA analysis) and the compositions of gut microbiota from the phylum and genus levels levels were not significant differences between the Boy and Girls groups when they were six months (Fig. 7B, 7E, 7H and 7k), one year (Fig. 7C, $7 \mathrm{~F}, 7 \mathrm{I}$ and $7 \mathrm{~L})$ and two years old (Fig. 7D, 7G, 7J and 7M) $(P>0.05)$, in which all above indicators were not significantly different between the caesarean (CB) and natural birth groups (NB) when they were six months (Fig. 8) $(P>0.05)$.

\section{Compositions of the gut microbiota in the breast milk and their correlations with the maternal characteristics}

As shown in Fig. 9, the percents of Proteobacteria (60.94 \pm 5.11$) \%$, Firmicutes $(23.81 \pm 4.30) \%$, Bacteroidetes $(9.56 \pm 2.98) \%$, Cyanobacteria $(1.36 \pm 0.61) \%$ and Actinobacteria $(0.76 \pm 0.12) \%$ constituted the dominant gut microbiota in the breast milk at the phylum level, which was accounted for more than $90 \%$ of the community (Fig. 9A and 9B). Meanwhile, Bacillus (3.59 \pm 2.99$) \%$, Sphingomonas (12.61 \pm 
4.64)\%, Staphylococcus $(4.52 \pm 2.21) \%$, unidentified_Alphaproteobacteria $(4.69 \pm 2.74) \%$, Brevundimonas $(9.52 \pm 2.40) \%$ and Streptococcus $(8.45 \pm 2.13) \%$ were the main dominant percents of the gut microbiota at the genus level in the breast milk (Fig. 9C, 9D and 9E). Then the associations between maternal characteristics (G, MA, M.BMI and I.BMI) and the gut microbiomes in the milk were discussed in Fig. $9 \mathrm{H}$, 9 I and Table 3, which were proved that the percents of Proteobacteria were negatively associated with the M.BMI and I.BMI. Meanwhile, there were significantly positive correlations between the I.BMI and the compositions of Firmicutes and Actinobacteria at the phylum level by the regression models (Fig. 9F) ( $P$ $<0.05$ ). From the genus level (Fig. 9G), the M.BMI was positively correlated with the percents of Sphingomonas and Bacillus, and negatively correlated with Limnobacter and Brevundimonas $(P<0.05)$. Meanwhile there was still positive significance between the composition of unidentified_Bacteroidales and I.BMI . 
Table 3

Correlations between the compositions of gut microbiota in the breast milk and the factors of maternal characteristics $(r, \mathrm{P})$

\begin{tabular}{|c|c|c|c|c|}
\hline & $\begin{array}{l}\text { Gestational } \\
\text { age }\end{array}$ & $\begin{array}{l}\text { Age of } \\
\text { pregnancy }\end{array}$ & $\begin{array}{l}\text { Pre- } \\
\text { pregnancy } \\
\text { BMI }\end{array}$ & $\begin{array}{l}\text { BMI } \\
\text { increment }\end{array}$ \\
\hline \multicolumn{5}{|l|}{ Phylum } \\
\hline Proteobacteria & $0.035,0.868$ & $0.004,0.984$ & $\begin{array}{l}-0.493 \\
0.012\end{array}$ & $-0.393,0.049$ \\
\hline Firmicutes & $0.005,0982$ & $-0.197,0.345$ & $0.237,0.253$ & $0.393,0.049$ \\
\hline Bacteroidetes & $0.129,0.540$ & $0.088,0.674$ & $0.238,0.252$ & $0.147,0.482$ \\
\hline Cyanobacteria & $-0.137,0.512$ & $0.302,0.142$ & $0.278,0.179$ & $-0.005,0.981$ \\
\hline Actinobacteria & $-0.058,0.783$ & $0.207,0.314$ & $0.206,0.323$ & $0.471,0.018$ \\
\hline Tenericutes & $-0.100,0.636$ & $0.086,0.682$ & $\begin{array}{l}-0.035 \\
0.869\end{array}$ & $0.203,0.331$ \\
\hline unidentified_Bacteria & $0.303,0.141$ & $-0.002,0.993$ & $0.365,0.073$ & $0.320,0.118$ \\
\hline Spirochaetes & $0.329,0.109$ & $0.092,0.661$ & $0.286,0.165$ & $0.245,0.237$ \\
\hline Melainabacteria & $0.278,0.179$ & $0.103,0.626$ & $0.178,0.395$ & $0.196,0.349$ \\
\hline Fibrobacteres & $-0.132,0.531$ & $0.131,0.532$ & $\begin{array}{l}-0.004 \\
0.984\end{array}$ & $0.213,0.307$ \\
\hline \multicolumn{5}{|l|}{ Genus } \\
\hline Bacillus & $0.057,0.789$ & $0.018,0.929$ & $0.398,0.047$ & $-0.054,0.796$ \\
\hline Sphingomonas & $-0.075,0.722$ & $-0.131,0.532$ & $\begin{array}{l}-0.312 \\
0.129\end{array}$ & $-0.272,0.189$ \\
\hline Staphylococcus & $0.128,0.543$ & $0.022,0.918$ & $0.309,0.133$ & $0.264,0.203$ \\
\hline unidentified_Alphaproteobacteria & $0.022,0.916$ & $0.134,0.523$ & $\begin{array}{l}-0.070 \\
0.740\end{array}$ & $0.108,0.606$ \\
\hline Brevundimonas & $-0.027,0.897$ & $-0.150,0.473$ & $\begin{array}{l}-0.480 \\
0.015\end{array}$ & $-0.112,0.595$ \\
\hline Streptococcus & $-0.002,0.992$ & $-0.167,0.424$ & $0.085,0.688$ & $0.162,0.439$ \\
\hline Limnobacter & $-0.004,0.984$ & $-0.153,0.466$ & $\begin{array}{l}-0.465 \\
0.019\end{array}$ & $-0.131,0.532$ \\
\hline Enhydrobacter & $-0.237,0.255$ & $-0.105,0.617$ & $0.036,0.866$ & $0.111,0.596$ \\
\hline Acinetobacter & $0.006,0.979$ & $0.002,0.991$ & $0.036,0.864$ & $-0.198,0.342$ \\
\hline
\end{tabular}




\begin{tabular}{|lllll|}
\hline & $\begin{array}{l}\text { Gestational } \\
\text { age }\end{array}$ & $\begin{array}{l}\text { Age of } \\
\text { pregnancy }\end{array}$ & $\begin{array}{l}\text { Pre } \\
\text { pregnancy } \\
\text { BMI }\end{array}$ & $\begin{array}{l}\text { BMI } \\
\text { increment }\end{array}$ \\
\hline Sphingobacterium & $0.255,0.219$ & $0.052,0.805$ & $\mathbf{0 . 4 0 0 , 0 . 0 3 7}$ & $-0.058,0.783$ \\
\hline Pseudomonas & $-0.136,0.518$ & $0.017,0.935$ & $\begin{array}{l}-0.203, \\
0.331\end{array}$ & $-0.032,0.878$ \\
\hline Lactobacillus & $-0.223,0.284$ & $-0.094,0.655$ & -0.273, & $0.239,0.251$ \\
\hline unidentified_Cyanobacteria & $-0.148,0.482$ & $0.278,0.178$ & $0.285,0.167$ & $-0.033,0.877$ \\
\hline Stenotrophomonas & $0.246,0.235$ & $-0.098,0.641$ & -0.004, & $-0.037,0.861$ \\
\hline unidentified_Bacteroidales & $0.032,0.878$ & $0.016,0.942$ & $0.032,0.880$ & $\mathbf{0 . 3 8 6 , 0 . 0 4 7}$ \\
\hline
\end{tabular}

\section{Discussion}

Our study showed how the gut microbiota developed during the two year of life after a normal term pregnancy in 30 full-term mother-infant pairs of exclusive breastfeeding within 6 months with the normal range of the growth and development index from birth to two years old. The results of this study proved that maternal (gut microbiota in the milk, pre-pregnancy BMI, BMI gain during the pregnancy) and infant characteristics (BMI at birth and increment of $\mathrm{BMI}$ ) were the key factors driving the assembly of an adultlike gut microbiota. Truly, we observed significant transitions in the infants'gut from the break milk, probably induced by the establishment of microbial interactions during community succession.

Based on the survey data of 16S rRNA, our results demonstrated there was an increased a-diversity and reduced $\beta$-diversity of gut microbiota in the stool samples of the growing infant from birth to two years old, pointing to the development of a more complex and less dissimilar microbiota over time, as reported previously of different population between the 6 months, one year and two years old children and maternal breast milk both compositionally and functionally, awaiting further maturation of the gut microbiota $[2,13]$. As the indicated above, the gut microbiota in the faeces might suffer from the sudden microbial succession phenomena and continue until the infants are 2 to 3 years old to reach a similar adult microbiota during the dynamic colonization, so we chose the different age subgroups (6-month, one-year and two-year) to discuss the dynamic colonization of the gut microbiota at the two year of life as the different food intake with the subsequent establishment and maintenance of the homeostasis of host microbiota during early life, events that may have immediate and long-term health consequences[14, 15]. Consistent with a previous study on the infants that Bifidobacterium, Streptococcus, Lactococcus, Lactobacillus were the dominate gut microbiota [16, 17], Besides, the gut microbiotas of 303 children from five Asian countries, namely Japan, China, Taiwan, Indonesia, and Thailand all found that they clustered into Bacteroides-Bifidobacterium-dominate microbiotas among the infants[18, 19]. Meanwhile, facultative anaerobic bacteria including the Staphylococcus, Streptococcus, Escherichia coli and 
Enterobacteria were thought to be the first colonizers of the gut, which was primarily changed to the phylum of Actinobacteria and Firmicutes following birth[20-22]. Around the age of one to two years old, the infant gut microbiome undergoes its second shift and the stable adult microbiome begins to emerge after that te weaning and solid foods were introduced[23]. Our results elucidated that the maturation of the gut microbiota was a nonrandom process of early life colonization connecting longitudinal data, where distinct interactions between the ten key microbial taxa (Bifidobacterium, Enterobacteriaceae, Klebsiella, Streptococcus, Clostridiales, Akkermansia, unidentified_Lachnospiraceae, Bacteroides, Faecalibacterium, Erysipelotrichaceae and Lachnoclostridium) could be identified at each sampled age. The different microbial configuration was also associated with functional shifts, as the increased capacity to degrade polysaccharides promoted by the introduction of solid foods. Indeed, recent research has shown that the certain bacteria might modulate the host pathways of Chemoheterotrophy, Fermentation, Parasites_or_symbionts, Nitrate_reduction and Aerobic_chemoheterotrophy metabolism[24].

As the previous studies, there were different host biology (gestational age at birth, mode of delivery, type of feeding and and gender) as the major driver for the dynamic process of gut microbiota by providing more detailed information about the timing of the bacterial identification beyond genus[24-26].

Therefore, these above influencing factors were all discussed by adjusting the breast-feeding until two year old in this study. Together with the development and colonization of an adult-like microbiota, our results also underscore the role of maternal and infant characteristics in the shaping and succession of gut microbial communities, in which there were significant associations between the gut microbiota in the milk, Pre-pregnancy BMI, BMI gain during the pregnancy, BMI at birth and increment of $\mathrm{BMI}$ among the infants, and the compositions of gut microbiota in the faeces. It is consistent with the previous studies[27-30], in which observed that most of the early colonizers are derived from the mother, while sharing of bacteria from skin and mouth was increased, in line with an earlier study. However, our results proved that no significant differences were shown between the gender and mode of productive subgroups, which is inconsistent with the original conclusions on metabolic and immune health that we are only beginning to understand[27, 31-33]. According to the analysis of the reasons for this inconsistency, the strength of this study was that all 30 spontaneous onset of labour at $39+$ weeks gestation with breastfeeding subjects were chosen according to the strict inclusion and exclusion criteria using repeated measurement data with the index of BMI, LAZ, WAZ and BMI Z between -1 and 1 at birth, 6 months, one year, one and a half year, and two years. Because there are recently some studies which have confirmed that gender and mode of productive style may affect the other adverse health outcomes[34-37]. It may be the cause of intestinal flora variation rather than the above variables itself.

\section{Conclusion}

In summary, the gut microbial community was significantly matured into adulthood in the increased age subgroups with the increasing trends of Firmicutes (Faecalibacterium, Blautia, Enterococcus, Subdoligranulum, Agathobacter, Unidentified_Erysipelotrichaceae, Staphylococcus, Acinetobacillus, Unidentified__Ruminococcaceae and Fusicatenibacter), Bacteroidetes and Verrucomicrobia, and 
decreasing trends of Actinobacteria (Bifidobacterium) and Proteobacteria (Enterobacteriaceae and Klebsiella) respectively at the phylum and genus levels, which revealed that certain bacteria might modulate the host pathways of Chemoheterotrophy, Fermentation, Parasites_or_symbionts, Nitrate_reduction and Aerobic_chemoheterotrophy metabolism. It also identified that there were significant correlations between the features of gut microbiota and maternal (gut microbiota in the milk, Pre-pregnancy BMI-M.BMI, BMI gain during the pregnancy-I.BMI) and infant characteristics (BMI at birthB.BMI and increment of BMI-G.BMI), which will provide a new direction for thost-gut microbiota interplay during the two year of life.

\section{Materials And Methods Study design}

A birth cohort was established with 254 healthy pregnant women and their infants who were recruited at the First Hospital of Tsinghua University from May to October 2017 according to the following strict inclusion and exclusion criteria. Specifically, we enrolled the subjects who were healthy at 20-40-year old without smoking history in gestation weeks of 6-12 among Chinese Han Population, intended to deliver in this hospital and routinely examined the growth and development related indicators within 2 years old of their infants. What is more, all infants should be full-term newborns and exclusively breastfed. The subjects were excluded if they had gastrointestinal diseases, gestational hypertension, hypothyroidism, diabetes, heart, liver, kidney or blood system related diseases, medical history of using antibiotics and thyroxine-related drugs during the pregnancy. Meanwhile, their infants with birth defects, genetic metabolic diseases, ischemia and hypoxia during the delivery were also excluded from this study.

This study protocol had been approved by the Ethics Committee of Beijing Children's Hospital, Capital Medical University (Registration number: 2016-20). All participants were clearly informed the purpose and significance of the survey by the investigator and signed informed consents. The clinical trial number of this study was recorded at the websites of http://www.chictr.org.cn/showproj.aspx?proj=4673. (Registration number: ChiCTR-OCH-14004900).

\section{Basic information questionnaires and biological sample collection}

The clinical information questionnaires, including the maternal age, height, pre-pregnancy weight, weight before delivery, gestational age and mode of delivery were collected by the trained investigators through face to face method when the participants were involved in this study. Meanwhile, the infant's information was used to obtain their diseases, drug usage, intake of the trace elements and the feeding methods at 6 month, one year, one and a half year, and two years they had the physical examination.

All biological sampling operations were executed in the morning by the trained professional researchers under the strict aseptic conditions and uniform protocols. Exactly, 5-10mL/sample breast milk was collected at the 3 months physical examination of their infants using the $15 \mathrm{~mL}$ sterile centrifuge tubes. 
Meanwhile, approximately $1 \mathrm{~g}$ stool samples were respectively obtained at 6 months (pure breast feeding in this period), one year (after solid food introduction), and two years (tend to the adults) physical examination of their infants using the sterile plastic spoons. Then all biological specimens were placed in the sterile tubes or vials, immediately frozen at $-20^{\circ} \mathrm{C}$, and then transported to the laboratory to store at $80^{\circ} \mathrm{C}$ until usage.

\section{Infant measures: Follow-up}

The anthropometric measurements of the infants were performed by the trained investigators at birth and each subsequent follow-up. The recumbent length and weight of the infants were determined to the nearest millimeter (HW-1000HW-2000, China) on a digital measuring board with three repeated measurements to calculate the mean value at birth, 6 months, one year, one and a half year, and two years, which were from the child welfare clinic and school health-care records in detail. The related BMI values $\left(\mathrm{kg} / \mathrm{m}^{2}\right)$ were calculated. Then the length for age $Z$ score (LAZ), weight for age $Z$ score (WAZ) and $\mathrm{BMI}$ for $\mathrm{Z}$ score (BMI Z) were calculated according to the growth curves under 5 years of age published by WHO in 2006 using the ANTHRO 2005 software downloaded from the WHO website (http://www.who.int/ childgrowth/ software/en/)[38, 39].

\section{Random selection of the subjects in this study}

According to the growth and development examining results at birth, 6 months, one year, one and a half year, and two years, 30 mothers-infant pairs were finally included as the subjects in this study with the normal range of the growth and development index (Fig. 1-BMI, LAZ, WAZ and BMI Z: -1 to 1) at birth, 6 months, one year, one and a half year, and two years. The basic information of the subjects was shown in Table 1.

\section{DNA preparation and High-throughput sequencing}

Within a strictly controlled, separate and sterile workplace, total DNA in the breast milk $(3 \mathrm{~mL})$ and faeces ( 200mg respectively at the 6 months, one year and two years) were approximately extracted using the QIAamp DNA Mini Kits (Qiagen, Valencia, CA). The isolated DNA samples were eluted in $50 \mu \mathrm{L}$ distilled water. Then the quality and quantity of each DNA sample were respectively measured by the agarose gel electrophoresis and Qubit 2.0 fluorometer (Life Technologies, Waltham, MA) before downstream processing. For each appropriate DNA sample, we amplified the variable regions 3 and 4 (V3-V4) in the 16S rRNA gene using the modified 515F (5'-GTGCCAGCMGCCGCGGTAA-3') and 806R (5'GGACTACNNGGGTATCTA AT-3') primers. After 16S rDNA library preparation and generation, the library quality was assessed on the Qubit 2.0 fluorometer. Then they were sequenced on the IlluminaHiSeq Platform 2500 and 250bp paired-end reads were generated at Novogene (Beijing, China).

\section{Statistical analysis}

The descriptive statistics were included the crude comparisons of the demographic factors and clinical parameters. The standard $P \leq 0.05$ was considered significant for all indicators analyses. The differences among all groups were tested by analysis of variance (ANOVA) for repeated measurement data and 
Kruskal-Wallis test based on whether the data was normally distributed, in which the compositions of intestinal flora were analyzed using the NovoMagic platform in the Beijing SinoGenoMax Center Co., Ltd (https://magic.novogene.com/customer/main\#/login). Exactly, the a-diversity indicators evaluating the

richness (Ace and Chao estimators) and diversity of the gut microbial community (Shannon and Simpson estimators) were calculated using the Faith phylo-genetic diversity. Principal coordinate analysis (PCA) and/or Non-Metric Multi-Dimensional Scaling (NMDS) analysis based on Bray-Curtis distance were performed to compare the compositions of the global microbiota at different age groups. Through LEfSe, network, ternaryplot and evolutionary_tree analysis, we could find the biomarker with statistical difference between all groups, then ANOVA (q test) was analyzed the significant differences at the phylum and genus levels. Meanwhile, the contribution of species to the differences was also quantified by the simper analysis. The Phylogenetic Investigation of Communities by Reconstruction of Unobserved States (PICRUSt) was chosen to predict the metagenome function using the 16S rRNA sequences and reference to the published genome sequences. The spear-man rank correlations and CCA/RDA analysis were used to measure the relationship between the environmental factors and microbial compositions at the phylum and genus levels.

\section{Abbreviations}

BMI

body mass index; LAZ:length for age Z score; WAZ:weight for age Z score; BMI Z:BMI for Z score; M.BMI:maternal pre-pregnancy BMI; I.BMI:BMI gain during the pregnancy; $\mathrm{B} . \mathrm{BMI}: \mathrm{BMI}$ at birth; S.BMI:BMI at 6 months old; O.BMI:BMI at one year old; T.BMI:BMI at two years old; G.BMl:increment of BMl; GM:gut microbioma in the breast milk; MA:maternal age; G:gestational week; ANOVA:analysis of variance; PICRUSt:phylogenetic investigation of communities by reconstruction of unobserved states; NMDS:nonmetric multi-dimensional scaling; PCA:principal coordinate analysis.

\section{Declarations}

\section{Acknowledgement}

We thank the infants and their mother for participating in this research. All authors express our thanks to YJL and YS for biological sample collection.

\section{Funding}

This study was supported by the Beijing Municipal Natural Science Foundation (7174302 to PL and S150006 to KQ) and the Research Funds of Profession Quota Budjet from Beijing Municipal Science and Technology Commission (\# bjsekyjs-2021 to KQ).

\section{Availability of data and materials}


We do not have unpublished data from the study. However, as soon as the paper is accepted, we would wish to make our results available to the scientific community. In addition, we would welcome collaboration with others. Our plan includes also presentations at national and international scientific meetings. In this study, important data might be posted and published on our institutional website.

\section{Authors' contributions}

PL and KMQ designed and supervised the study. YS and YJL managed the clinical research. XLC and XYC obtained the samples and clinical details. TTT performed 16S rRNA sequencing of the microbiota. CXL and PL performed the data analysis. PL assisted in statistical analysis of the metadata. PL and KMQ wrote the manuscript. All authors read and approved the final manuscript.

\section{Ethics approval and consent to participate}

The study was committed at Beijing Pediatric Research Institution, Beijing Children's Hospital『Capital Medical University, National Center for Children's Health. And this clinical trial registry number was approved by the websites of http://www.chictr.org.cn/showproj.aspx?proj=4673 (ChiCTR-OCH14004900). Detail has been removed from these case descriptions to ensure anonymity. While the informed consent was obtained from all subjects.

\section{Consent for publication}

Not applicable.

\section{Competing interests}

The authors declare that they have no competing interests.

\section{Authors' information}

${ }^{1}$ Laboratory of Nutrition and Development, Key Laboratory of Major Diseases in Children Ministry of Education, Beijing Pediatric Research Institution, Beijing Children's Hospital区Capital Medical University, National Center for Children's Health, Beijing, China. ${ }^{2}$ Child Health-care Department, Chaoyang District Maternal and Child Health Care Hospital of Beijing, Beijing, China. ${ }^{3}$ Child Health-care Department, Shunyi District Maternal and Child Health Care Hospital of Beijing, Beijing, China.

\section{References}

1. Martin R, et al. Early-Life Events, Including Mode of Delivery and Type of Feeding, Siblings and Gender, Shape the Developing Gut Microbiota. PLoS One. 2016;11(6):e0158498.

2. Bäckhed F, et al. Dynamics and Stabilization of the Human Gut Microbiome during the First Year of Life. Cell Host Microbe. 2015;17(5):690-703. 
3. Xiong X, et al. Modelling the effect of birth and feeding modes on the development of human gut microbiota. Proc Biol Sci. 2021; 288(1942): 20201810.

4. Korpela K, et al. Maternal Fecal Microbiota Transplantation in Cesarean-Born Infants Rapidly Restores Normal Gut Microbial Development: A Proof-of-Concept Study. Cell. 2020;183(2):324-34.

5. Milani C, et al. The First Microbial Colonizers of the Human Gut: Composition, Activities, and Health Implications of the Infant Gut Microbiota. Microbiol Mol Biol Rev. 2017;81(4):e00036-17.

6. Jing $L$, et al. Complementary feeding pattern and its effect on nutritional status among infants. Chinese Journal of Food Hygiene. 2021;33(3):391-6.

7. Ronan V, Yeasin R, Claud EC. Childhood Development and the Microbiome-The Intestinal Microbiota in Maintenance of Health and Development of Disease During Childhood Development. Gastroenterology. 2021;160(2):495-506.

8. Vandenplas $\mathrm{Y}$, et al. Factors affecting early-life intestinal microbiota development. Nutrition. 2020;78:110812.

9. Pannaraj PS, et al. Association Between Breast Milk Bacterial Communities and Establishment and Development of the Infant Gut Microbiome. JAMA Pediatr. 2017;171(7):647-54.

10. Rutayisire $E$, et al. The mode of delivery affects the diversity and colonization pattern of the gut microbiota during the first year of infants' life: a systematic review. BMC Gastroenterol. 2016;16(1):86.

11. Rosenberg E, et al. The evolution of animals and plants via symbiosis with microorganisms. Environ Microbiol Rep. 2010;2(4):500-6.

12. Lim ES, Wang D, Holtz LR. The Bacterial Microbiome and Virome Milestones of Infant Development. Trends Microbiol. 2016;24(10):801-10.

13. Stanislawski MA, et al. Pre-pregnancy weight, gestational weight gain, and the gut microbiota of mothers and their infants. Microbiome. 2017;5(1):113.

14. Fouhy F, et al. Perinatal factors affect the gut microbiota up to four years after birth. Nat Commun. 2019;10(1):1517.

15. Yatsunenko T, et al. Human gut microbiome viewed across age and geography. Nature. 2012;486(7402):222-7.

16. La Rosa PS, et al. Patterned progression of bacterial populations in the premature infant gut. Proc Natl Acad Sci U S A. 2014;111(34):12522-7.

17. Stokholm J, et al. Maturation of the gut microbiome and risk of asthma in childhood. Nat Commun. 2018;9(1):141.

18. Nakayama J, et al. Diversity in gut bacterial community of school-age children in Asia. Sci Rep. 2015;5:8397.

19. Tanaka M, Nakayama J. Development of the gut microbiota in infancy and its impact on health in later life. Allergol Int. 2017;66(4):515-22. 
20. Turroni F, et al. Diversity of bifidobacteria within the infant gut microbiota. PLoS One. 2012;7(5):e36957.

21. Voreades N, Kozil A, Weir TL. Diet and the development of the human intestinal microbiome. Front Microbiol. 2014;5:494.

22. Bergström A, et al. Establishment of intestinal microbiota during early life: a longitudinal, explorative study of a large cohort of Danish infants. Appl Environ Microbiol. 2014;80(9):2889-900.

23. Penders $\mathrm{J}$, et al. Establishment of the intestinal microbiota and its role for atopic dermatitis in early childhood. J Allergy Clin Immunol. 2013;132(3):601-7.

24. Kim H, et al. Birth Mode, Breastfeeding, Pet Exposure, and Antibiotic Use: Associations With the Gut Microbiome and Sensitization in Children. Curr Allergy Asthma Rep. 2019;19(4):22.

25. Zhou $X$, et al. Early-life food nutrition, microbiota maturation and immune development shape lifelong health. Crit Rev Food Sci Nutr. 2019;59(sup1):30-8.

26. Nogacka AM, et al. Early microbiota, antibiotics and health. Cell Mol Life Sci. 2018;75(1):83-91.

27. Baumann-Dudenhoeffer AM, et al. Infant diet and maternal gestational weight gain predict early metabolic maturation of gut microbiomes. Nat Med. 2018;24(12):1822-9.

28. Hara A, Iwasa Y. Coupled dynamics of intestinal microbiome and immune system-A mathematical study. J Theor Biol. 2019;464:9-20.

29. Macpherson AJ, de Agüero MG, Ganal-Vonarburg SC. How nutrition and the maternal microbiota shape the neonatal immune system. Nat Rev Immunol. 2017;17(8):508-17.

30. Shao Y, et al. Stunted microbiota and opportunistic pathogen colonization in caesarean-section birth. Nature. 2019;574(7776):117-21.

31. Ding T, Schloss PD. Dynamics and associations of microbial community types across the human body. Nature. 2014;509(7500):357-60.

32. Ardeshir A, et al. Breast-fed and bottle-fed infant rhesus macaques develop distinct gut microbiotas and immune systems. Sci Transl Med. 2014;6(252):252ra120.

33. Mueller NT, et al. Prenatal exposure to antibiotics, cesarean section and risk of childhood obesity. Int J Obes (Lond). 2015;39(4):665-70.

34. Society for Adolescent Health and Medicine. Promoting Health Equality and Nondiscrimination for Transgender and Gender-Diverse Youth. J Adolesc Health. 2020;66(6):761-5.

35. Aghai ZH, et al. Gender variations in neonatal and early infant mortality in India and Pakistan: a secondary analysis from the Global Network Maternal Newborn Health Registry. Reprod Health. 2020;17(Suppl 3):178.

36. Arboleya S, et al. C-section and the Neonatal Gut Microbiome Acquisition: Consequences for Future Health. Ann Nutr Metab. 2018;73(Suppl 3):17-23.

37. Levin ME, et al. Environmental factors associated with allergy in urban and rural children from the South African Food Allergy (SAFFA) cohort. J Allergy Clin Immunol. 2020;145(1):415-26. 
38. Fukase K, et al. Effect of eradication of Helicobacter pylori onincidence of metachronous gastric carcinoma after endoscopic resection of earlygastric cancer: an open-label, randomised controlled trial. Lancet. 2008;372(9636):392-7.

39. World Health Organization. WHO Multicentre Growth Reference Study Group. WHO child growth standards: length/height-for-age, weight-for-age, weight-for-length, weight-for-height and body mass index-for-age: Methods and development. Geneva: WHO; 2007.

\section{Figures}
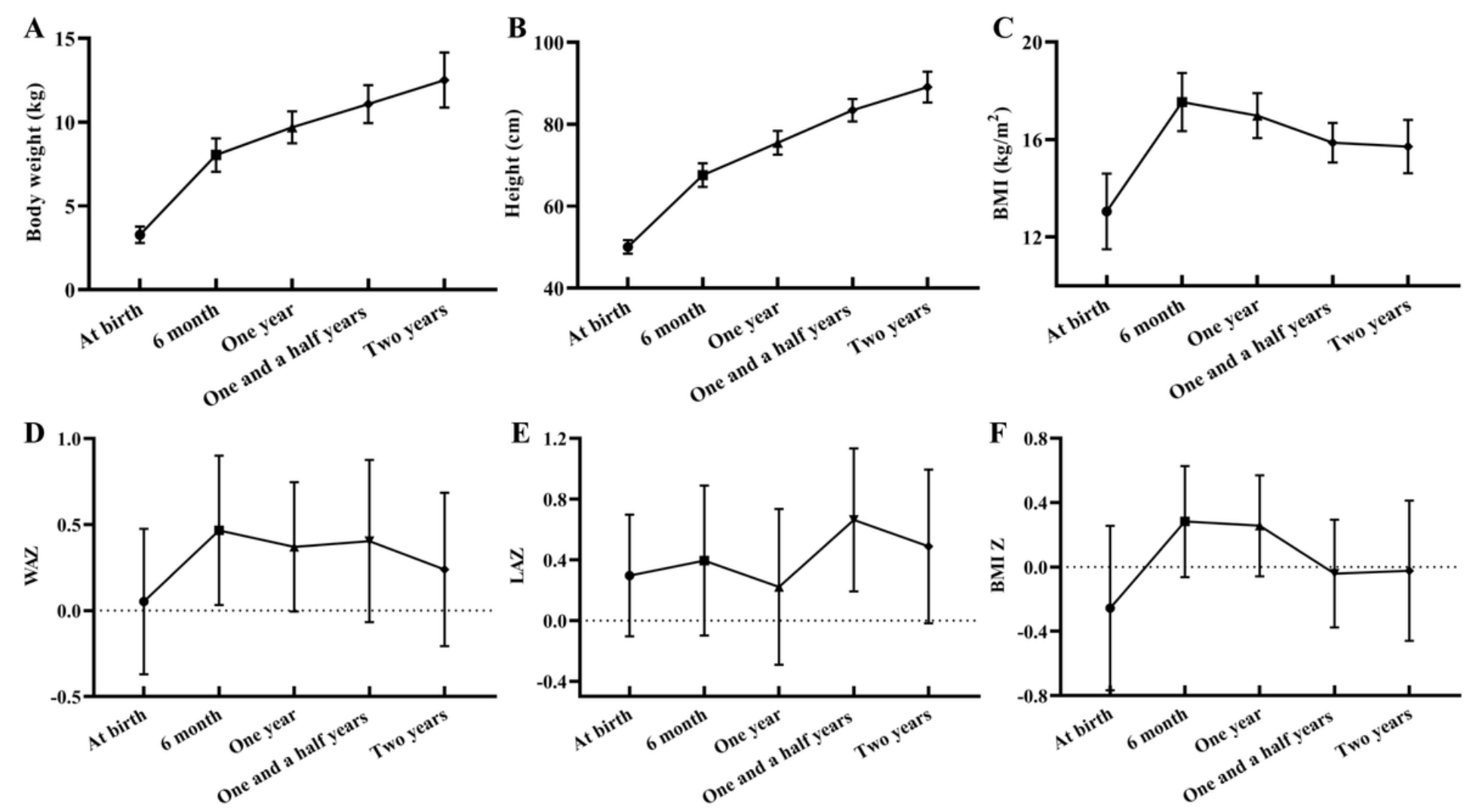

Figure 1

Characteristics of growth and development related indicators among the infants at different ages from birth to two years old. A: Body weight at birth, 6-month, one-year, one and a half year and two-year old. B: Height at birth, 6-month, one-year, one and a half year and two-year old. C: BMI at birth, 6-month, one-year, one and a half year and two-year old. D: WAZ at birth, 6-month, one-year, one and a half year and two-year old. E: LAZ at birth, 6-month, one-year, one and a half year and two-year old. F: BMI Z at birth, 6-month, one-year, one and a half year and two-year old. Note: BMI: Body mass index, WAZ: Weight for age Z score, LAZ: Length for age Z score, BMI Z: BMI for age Z score. 

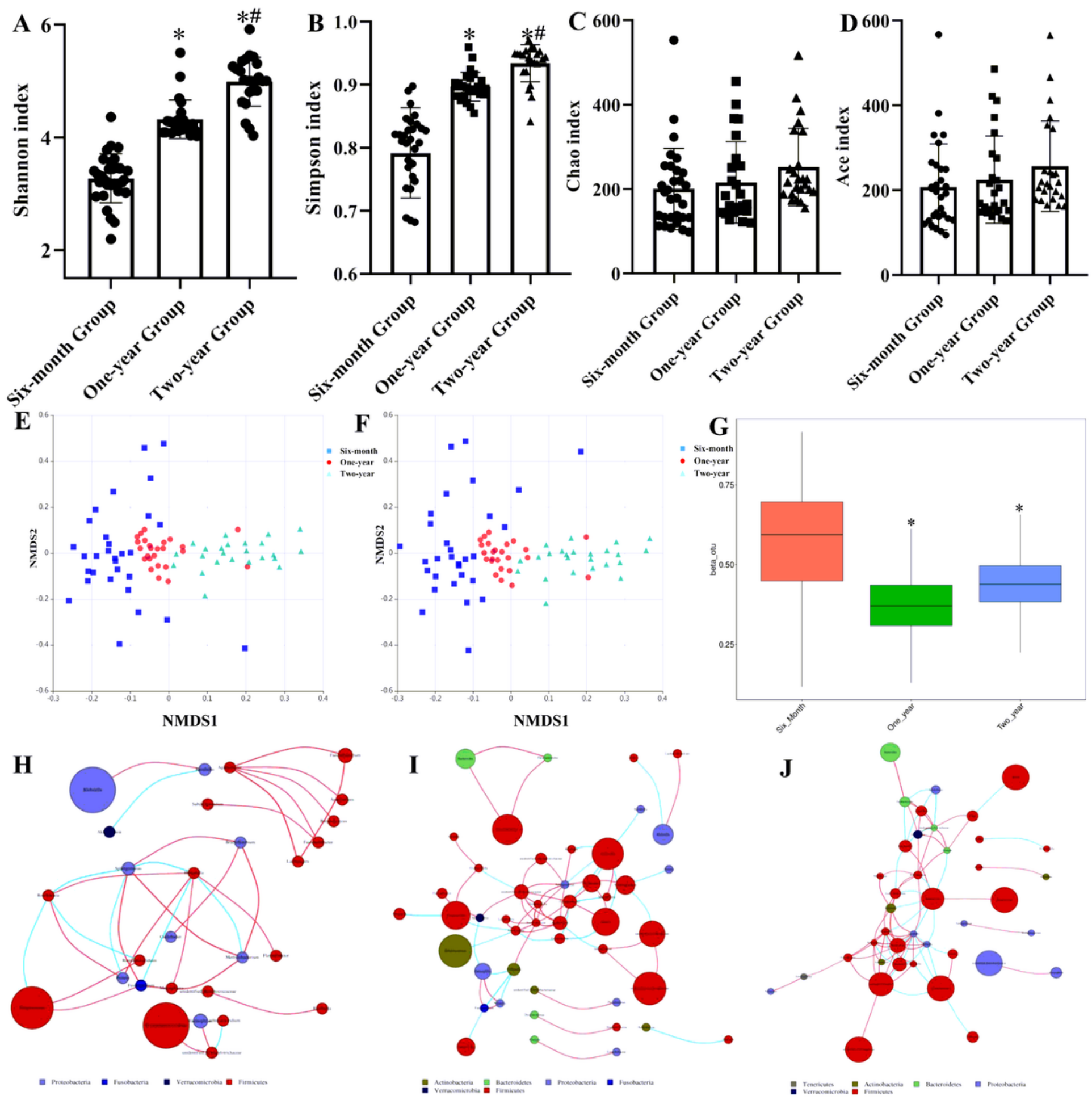

Figure 2

The diversity of gut microbiota at different ages from birth to two years old. A: Shannon index in sixmonth, one-year and two-year groups. B: Simpson index in six-month, one-year and two-year groups. C: Chao index in six-month, one-year and two-year groups. D: Ace index in six-month, one-year and two-year groups. E: Non-metric multidimensional scaling (NMDS) analysis at the phylum level in six-month, oneyear and two-year groups. F: NMDS analysis at the genus level in six-month, one-year and two-year groups. G: The average of $\beta$ diversity in six-month, one-year and two-year groups. $\mathrm{H}$ : The network analysis 
of gut microbiota at six month from the phylum and genus levels. I: The network analysis of gut microbiota at one year from the phylum and genus levels. J: The network analysis of gut microbiota at two years from the phylum and genus levels. Note: ${ }^{*}$ Compared to the six-month group, $\mathrm{P}<0.05$; \# Compared to the one-year group, $\mathrm{P}<0.05$.
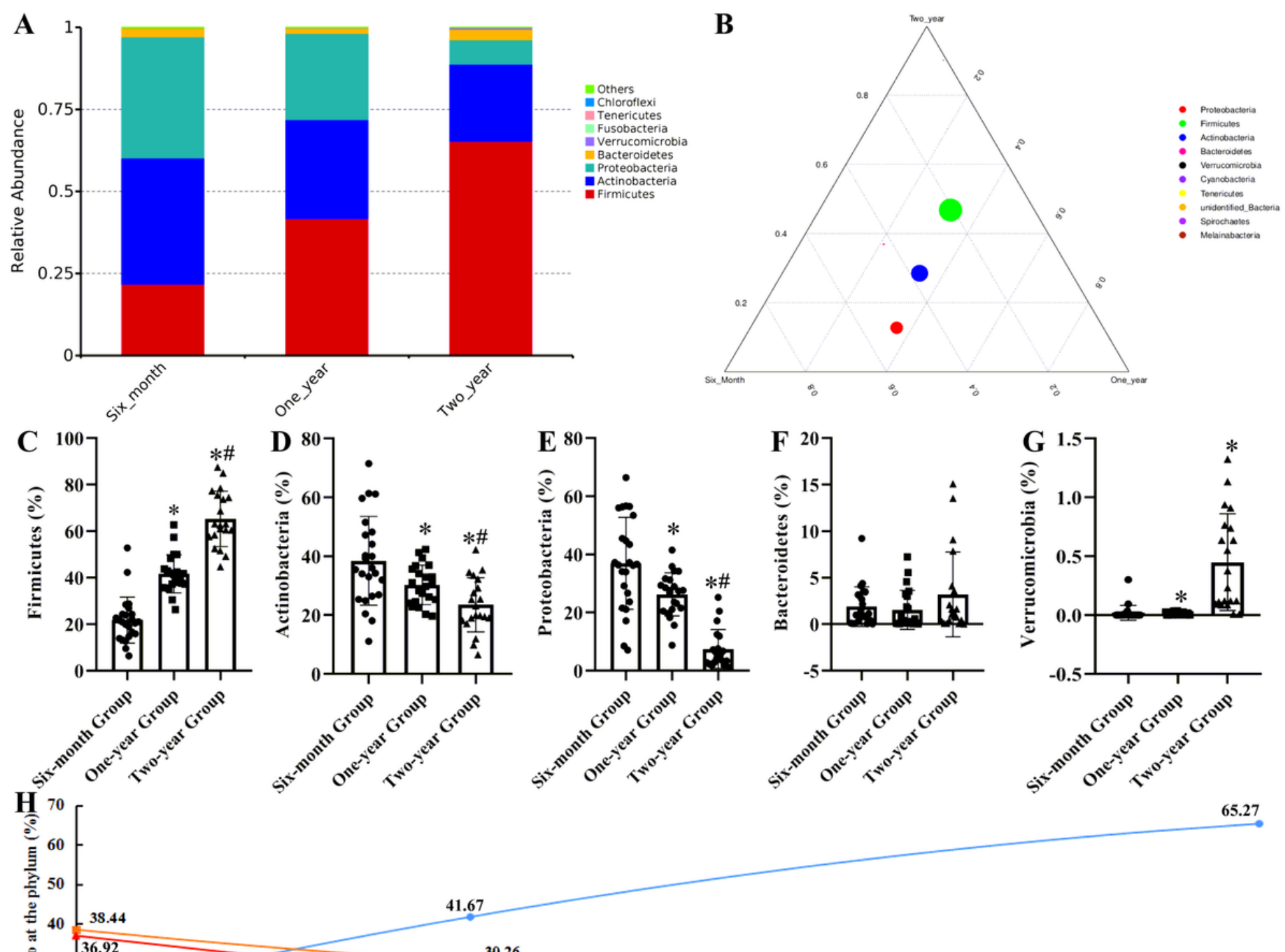

65.27

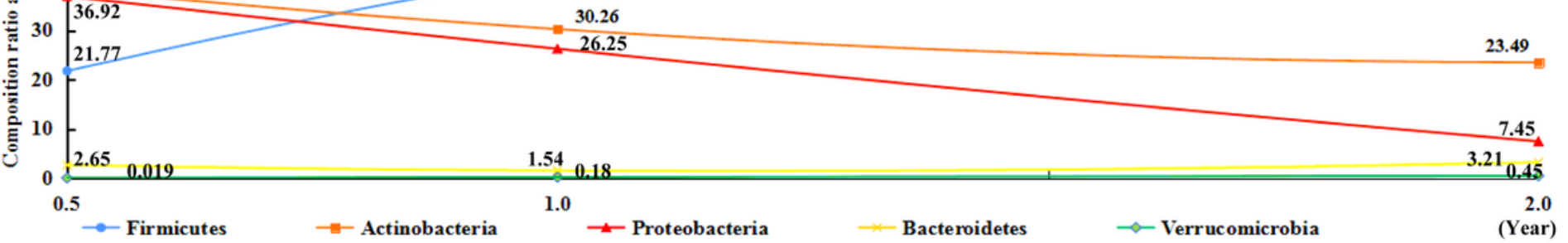

Figure 3

The compositions of gut microbiota among the infants at the phylum level from birth to two years old. A: The relative top eight abundance of gut microbiota in six-month, one-year and two-year groups. $\mathrm{B}$ : The ternary-plot analysis of gut microbiota in all groups. C: The percent of Firmicutes in all groups. D: The percent of Actinobacteria in all groups. E: The percent of Proteobacteria in all groups. F: The percent of Bacteroidetes in all groups. G: The percent of Verrucomicrobia in all groups. $\mathrm{H}$ : The composition trends of 
gut microbiota from birth to two years old. ${ }^{*}$ Compared to the six-month group, $\mathrm{P}<0.05$; \# Compared to the one-year group, $\mathrm{P}<0.05$.
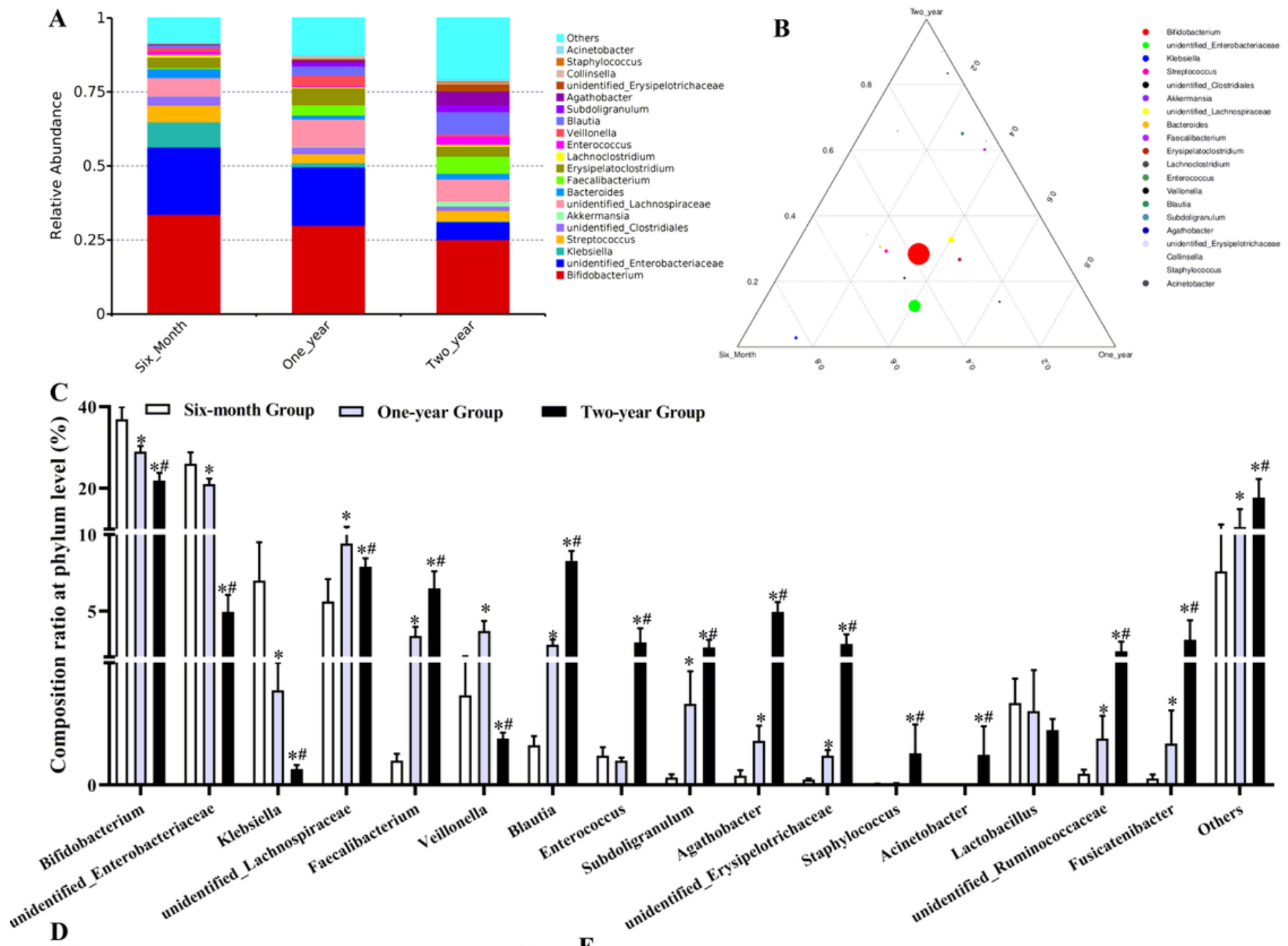

D
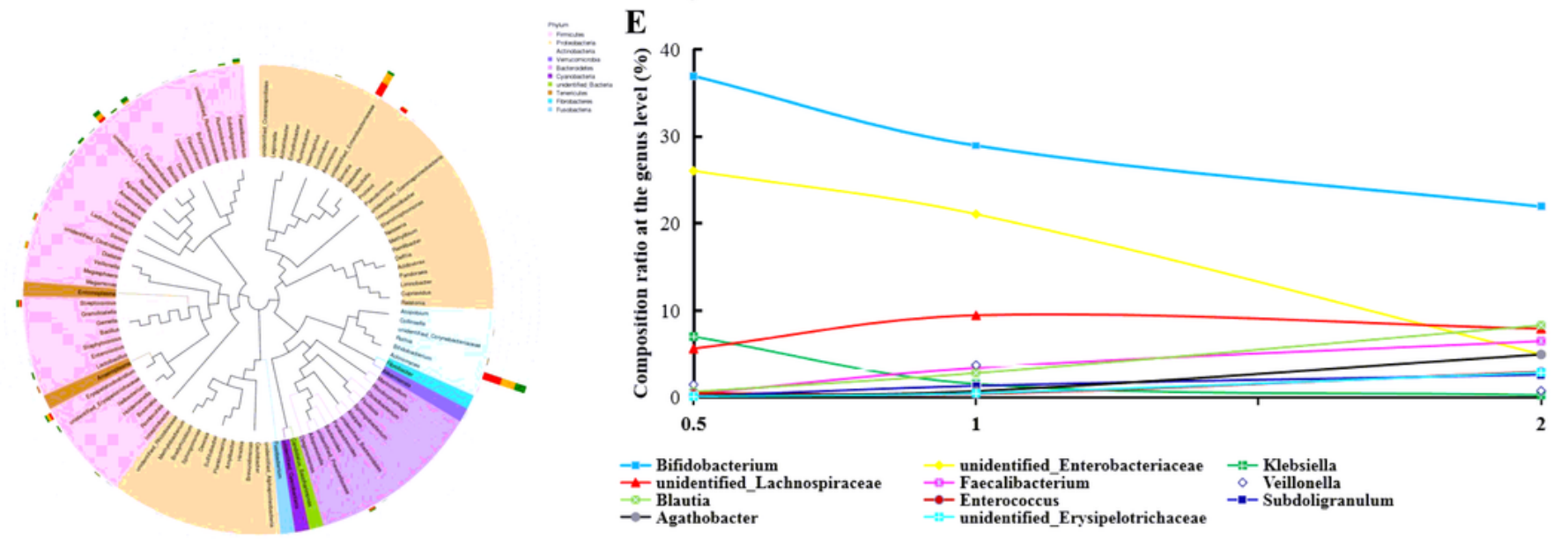

Figure 4

The compositions of gut microbiota among the infants at the genus level from birth to two years old. A: The relative top twenty abundance of gut microbiota in six-month, one-year and two-year groups. B: The ternaryplot analysis of gut microbiota in six-month, one-year and two-year groups. C: The compositions 
of gut microbiota in six-month, one-year and two-year groups. D: The evolutionary tree of gut microbiota in all groups. E: The composition trends of gut microbiota in all groups. ${ }^{*}$ Compared to the six-month group, $\mathrm{P}<0.05$. \# Compared to the one-year group, $\mathrm{P}<0.05$.
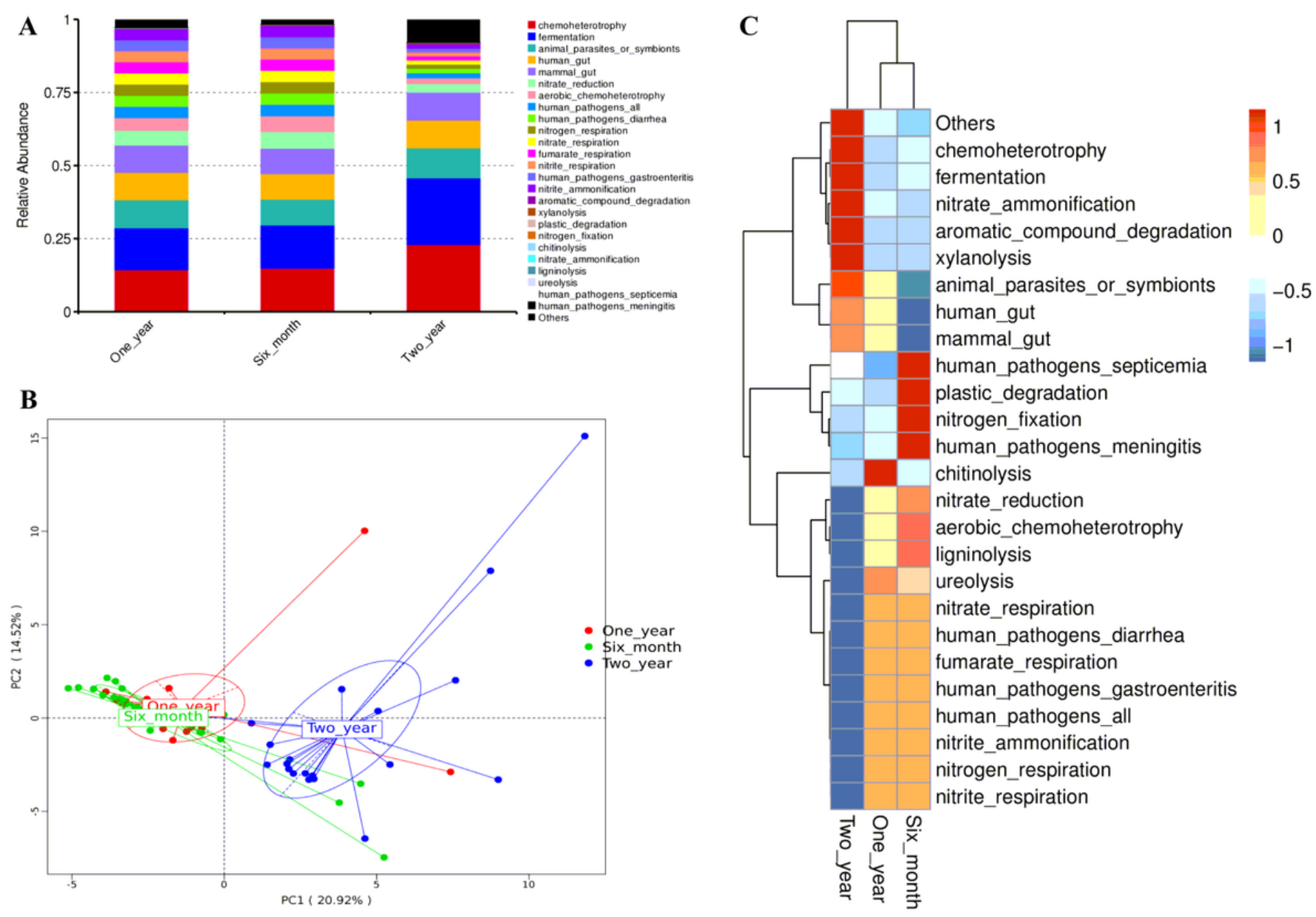

Figure 5

The microbial functional features among the infants in different ages from birth to two years old. A: The KEGG (Kyoto Encyclopedia of Genes and Genomes) database pathways. B: The PCA analysis of microbial functional features in different groups. C: The microbial functional features in different ages from birth to two years old among the infants. 

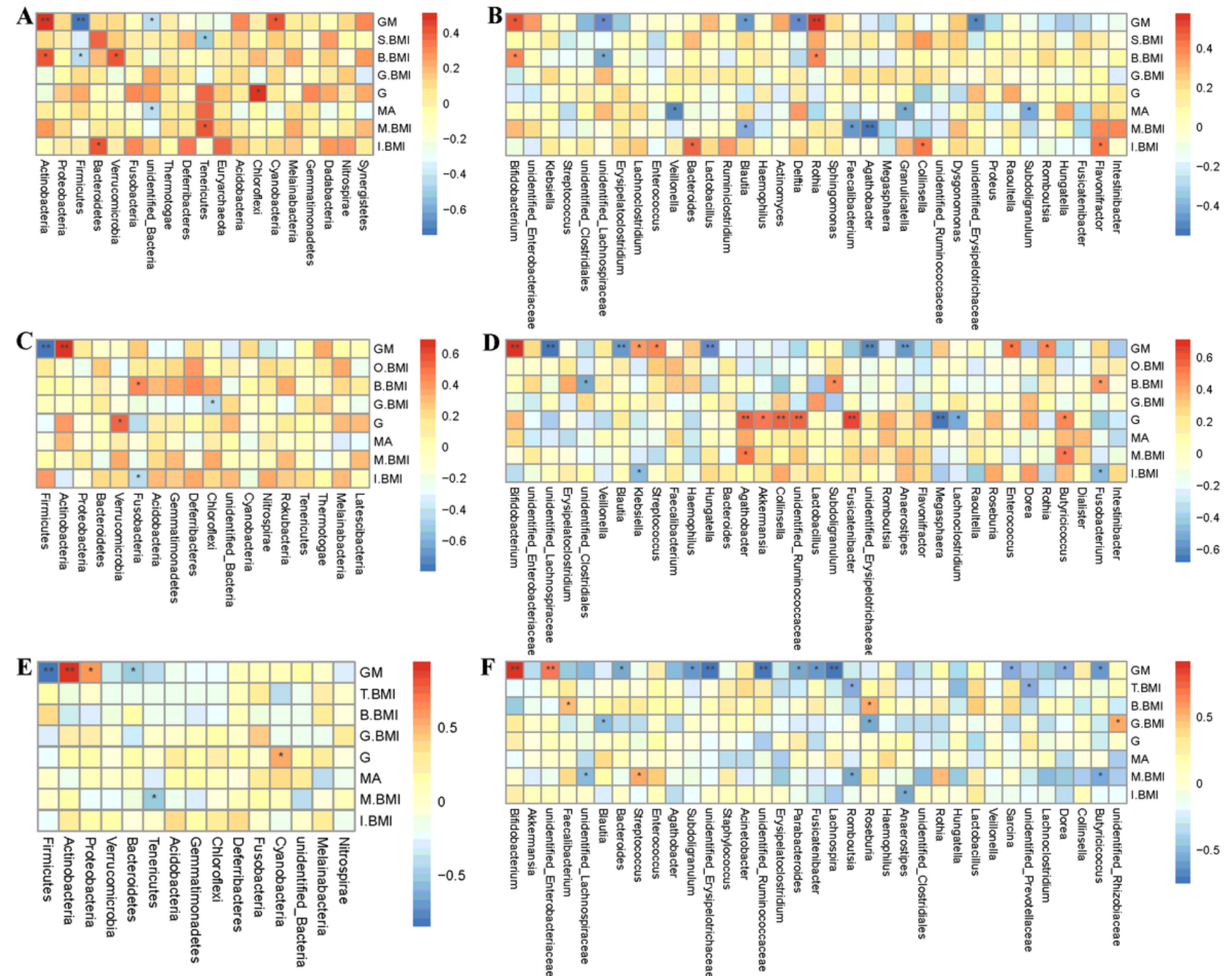

$\mathbf{F}$
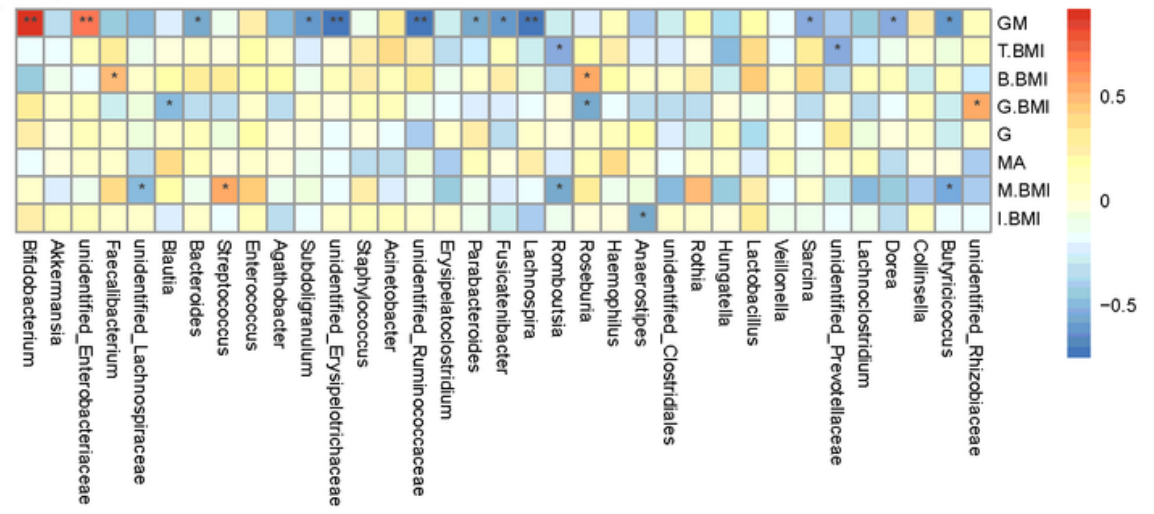

\section{Figure 6}

Correlations between maternal (infant) characteristics and the compositions of gut microbiota at the phylum and genus levels in different ages from birth to two years old A: Correlations between maternal and infant characteristics, and the compositions of gut microbiota at the phylum level among the infants of six month old. B: Correlations between maternal and infant characteristics, and the compositions of gut microbiota at the genus level among the infants of six month old. C: Correlations between maternal and infant characteristics, and the compositions of gut microbiota at the phylum level among the infants of one year old. D: Correlations between maternal and infant characteristics, and the compositions of gut microbiota at the genus level among the infants of one year old. E: Correlations between maternal and infant characteristics, and the compositions of gut microbiota at the phylum level among the infants of two years old. F: Correlations between maternal and infant characteristics, and the compositions of gut microbiota at the genus level among the infants of two years old. Note: GM: Compositions of gut microbiota in the breast milk, S.BMI: BMI at six month old, O.BMI: BMI at one year old, T.BMI: BMI at two 
years old, B.BMI: BMI at birth, G.BMI: BMI growth, G: Gestational age, MA: Mother age at the pregnancy, M.BMI: Maternal BMI at the stage of pre-pregnancy, I.BMI: BMI increment during the pregnancy, *represented that the correlations were statistically significant, $\mathrm{P}<0.05$.
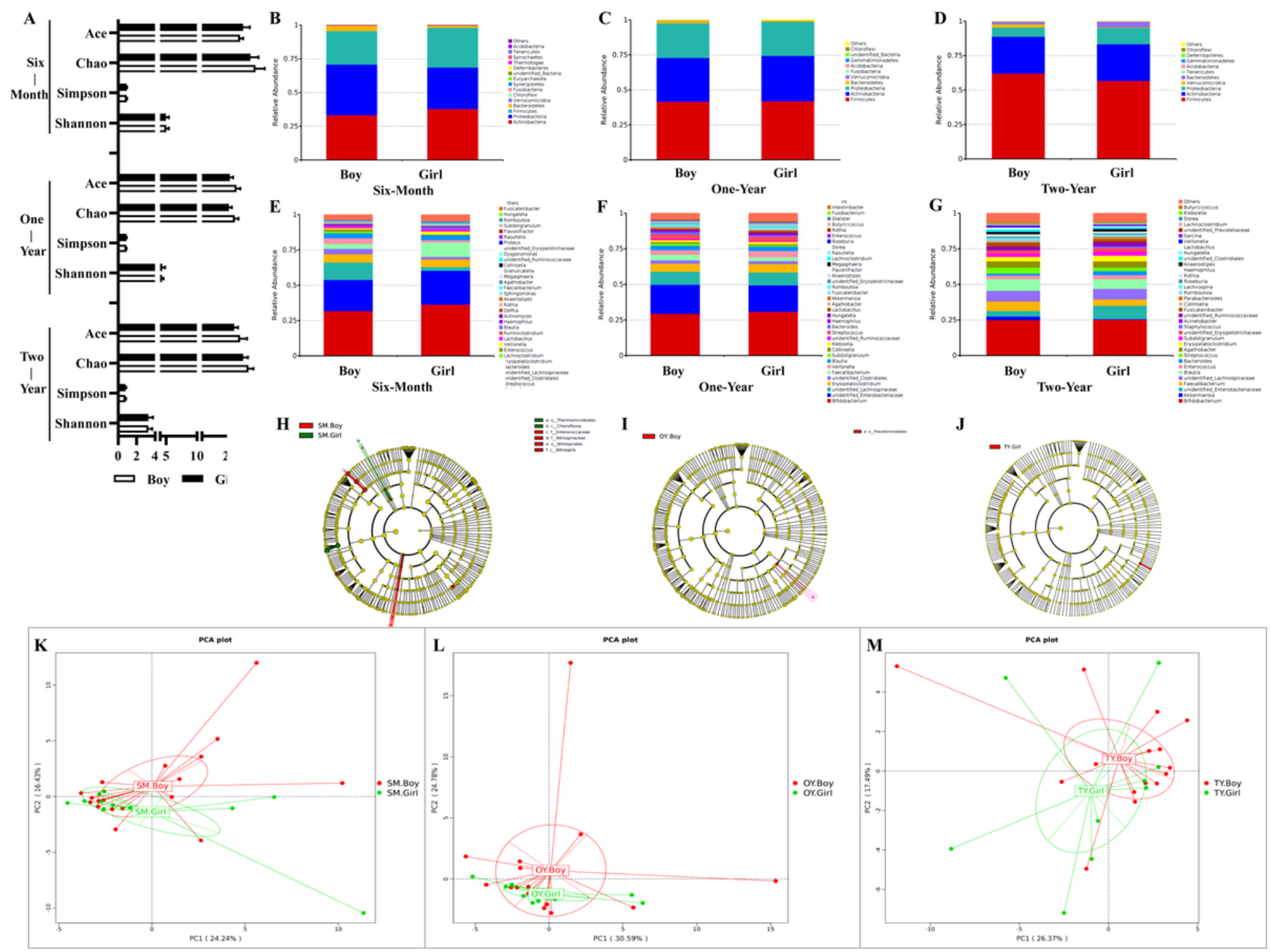

\section{Figure 7}

The diversity and compositions of gut microbiota in different sex subgroups among the infants with different ages. A: The a diversity index (Shannon, Simpson, Chao and ACE index) between the boy and girl subgroups. B: Compositions of gut microbiota at the phylum level between the boy and girl subgroups among the infants of six month old. C: Compositions of gut microbiota at the phylum level between the boy and girl subgroups among the infants of one year old. D: Compositions of gut microbiota at the phylum level between the boy and girl subgroups among the infants of two years old. E: Compositions of gut microbiota at the genus level between the boy and girl subgroups among the infants of six month old. F: Compositions of gut microbiota at the genus level between the boy and girl subgroups among the infants of one year old. G: Compositions of gut microbiota at the genus level between the boy and girl subgroups among the infants of two years old. $\mathrm{H}$ : Cladogram analysis of gut microbiota at the genus level between the boy and girl subgroups among the infants of six month old. I: 
Cladogram analysis of gut microbiota at the genus level between the boy and girl subgroups among the infants of one year old. J: Cladogram analysis of gut microbiota at the genus level between the boy and girl subgroups among the infants of two years old. K: PCA analysis of gut microbiota at the genus level between the boy and girl subgroups among the infants of six month old. L: PCA analysis of gut microbiota at the genus level between the boy and girl subgroups among the infants of one year old. M: PCA analysis of gut microbiota at the genus level between the boy and girl subgroups among the infants of two years old
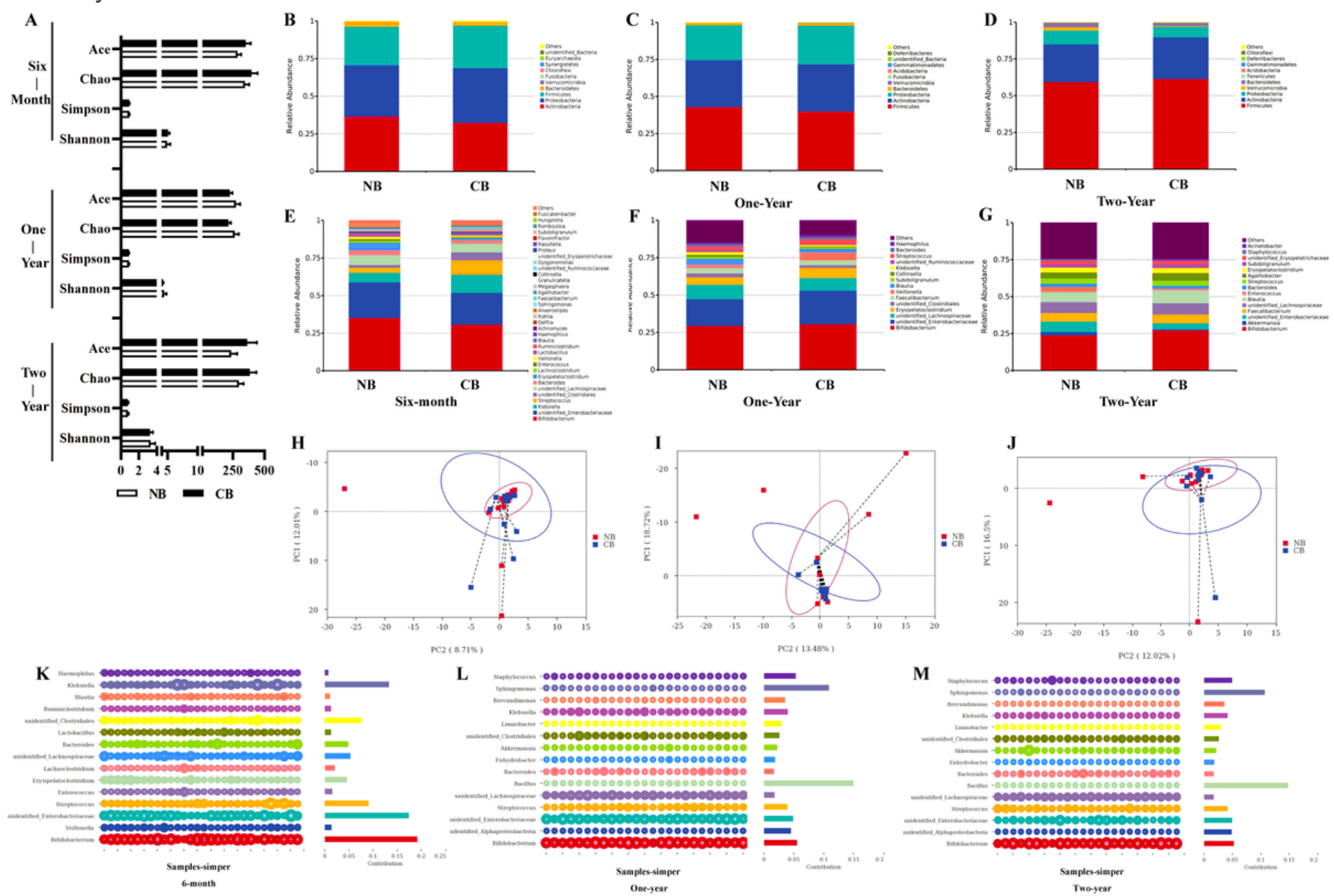

Figure 8

The diversity and compositions of gut microbiota in different productive mode subgroups among the infants with different ages. A: The a diversity index (Shannon, Simpson, Chao and ACE index) between the natural (NB) and caesarean birth (CB) subgroups. B: Compositions of gut microbiota at the phylum level between NB and CB subgroups among the infants of six month old. C: Compositions of gut microbiota at the phylum level between NB and CB subgroups among the infants of one year old. D: Compositions of gut microbiota at the phylum level between NB and CB subgroups among the infants of two years old. E: Compositions of gut microbiota at the genus level between NB and CB subgroups among the infants of six month old. F: Compositions of gut microbiota at the genus level between NB and CB subgroups among the infants of one year old. G: Compositions of gut microbiota at the genus 
level between NB and CB subgroups among the infants of two years old. H: PCA analysis of gut microbiota at the genus level between NB and CB subgroups among the infants of six month old. I: PCA analysis of gut microbiota at the genus level between NB and CB subgroups among the infants of one year old. J: PCA analysis of gut microbiota at the genus level between NB and CB subgroups among the infants of two years old. K: Simple analysis of gut microbiota at the genus level between NB and CB subgroups among the infants of six month old. L: Simple analysis of gut microbiota at the genus level between NB and CB subgroups among the infants of one year old. M: Simple analysis of gut microbiota at the genus level between NB and CB subgroups among the infants of two year old.

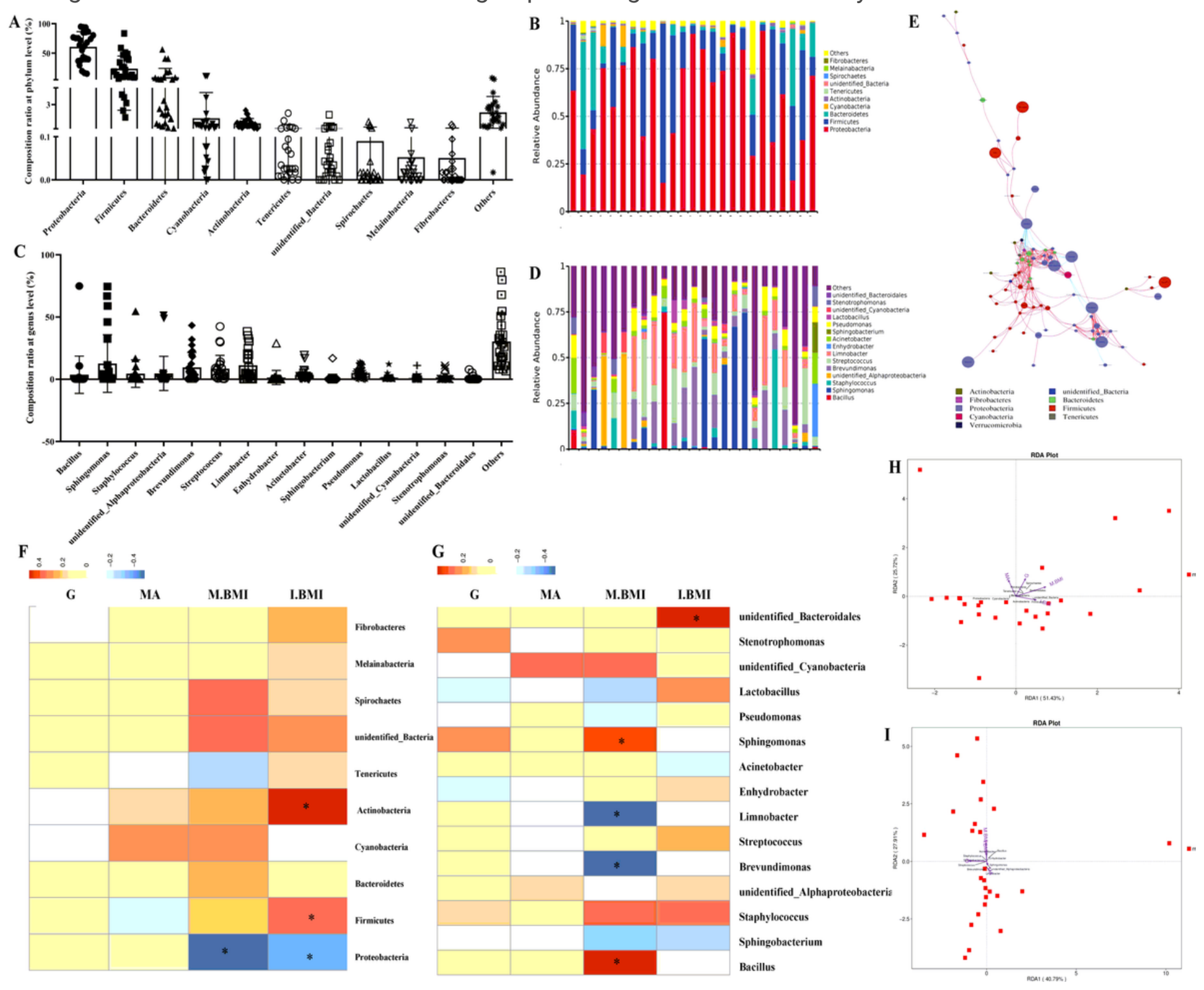

\section{Figure 9}

Compositions of gut microbiota in the breast milk and their correlations with the maternal characteristics A and B: Compositions of gut microbiota at the phylum level. C and D: Compositions of gut microbiota at the genus level. E: The network analysis of gut microbiota at the phylum and genus levels. F: Correlations between maternal characteristics and the compositions of gut microbiota at the phylum level. G: 
Correlations between maternal characteristics with the compositions of gut microbiota at the genus level. $\mathrm{H}$ : RDA analysis of gut microbiota at the phylum level. I: RDA analysis of gut microbiota at the genus level. Note: G: Gestational age, MA: Mother age at the pregnancy, M.BMI: Maternal BMI at the stage of pre-pregnancy, I.BMI: BMI increment during the pregnancy, *represented that the correlations between the compositions of gut microbiota in the breast milk and maternal characteristics were statistically significant, $\mathrm{P}<0.05$. 